\title{
Some Classes of Operators Related to p-Hyponormal Operator
}

\author{
Md. Ilyas ${ }^{1}$, Reyaz Ahmad ${ }^{2}$ \\ ${ }^{1}$ Department of Mathematics, Gaya College, Gaya, Bihar, India \\ ${ }^{2}$ Al-Ain University of Science and Technology, Al Ain \& Abu Dhabi, UAE \\ Email: reyaz56@hotmail.com, reyaz56@gmail.com
}

Received August 11, 2012; revised September 16, 2012; accepted September 28, 2012

\begin{abstract}
We introduce a new family of classes of operators termed as ${ }^{*} p$-paranormal operator, classes ${ }^{*} A(p, p) ; p>0$ and ${ }^{*} A(p, q) ; p, q>0$, parallel to $p$-paranormal operator and classes $A(p, p) ; p>0$ and $A(p, q) ; p, q>0$ introduced by M. Fujii, D. Jung, S. H. Lee, M. Y. Lee and R. Nakamoto [1]. We present a necessary and sufficient condition for p-hyponormal operator $T \in B(H)$ to be ${ }^{*} p$-paranormal and the monotonicity of ${ }^{*} A(p, q)$. We also present an alternative proof of a result of M. Fujii, et al. [1, Theorem 3.4].
\end{abstract}

Keywords: $p$-Hyponormal Operator; Monotonicity; Class of Operators * $A(p, q)$; 'Paranormal Operator; ${ }^{*} p$-Paranormal Operator

\section{Introduction}

Let $B(H)$ denote the algebra of bounded liner operators on a Hilbert space $H$. An operator $T \in B(H)$ is positive if $(T x, x) \geq 0$ for all $x \in H$. An operator $T \in B(H)$ is hyponormal if $T^{*} T \geq T T^{*}$ and $p$-hyponormal if $\left(T^{*} T\right)^{p} \geq\left(T T^{*}\right)^{p}$ for $p>0$. By the well known Lowner-Heinz theorem " $A \geq B \geq 0$ ensures $A^{\alpha} \geq B^{\alpha}$ for $\alpha \in(0,1)$ ", every $p$-hyponormal operator is $q$-hyponormal for $p \geq q \geq 0$. The Furuta's inequalities [2] are as follows:

If $A \geq B \geq 0$ then for each $r_{0} \geq 0$

$$
\begin{aligned}
& \left(B^{r_{0}} A^{p_{0}} B^{r_{0}}\right)^{1 / q_{0}} \geq\left(B^{r_{0}} B^{p_{0}} B^{r_{0}}\right)^{1 / q_{0}} \\
& \left(A^{r_{0}} A^{p_{0}} A^{r_{0}}\right)^{1 / q_{0}} \geq\left(A^{r_{0}} B^{p_{0}} A^{r_{0}}\right)^{1 / q_{0}}
\end{aligned}
$$

hold for $p_{0} \geq 0$ and $q_{0} \geq 1$ with $\left(1+2 r_{0}\right) q_{0} \geq p_{0}+2 r_{0}$.

An operator $T \in B(H)$ is

1) paranormal if $\left\|T^{2} x\right\|\|x\| \geq\|T x\|^{2}$ for all $x \in H$;

2) " paranormal if $\left\|T^{2} x\right\|\|x\| \geq\left\|T^{*} x\right\|^{2}$ for all $x \in H$.

\section{Preliminaries and Background}

M. Fujii, D. Jung, S. H. Lee, M. Y. Lee and R. Nakamoto [1] introduced the following classes of operators:

An operator $T \in B(H)$ is $p$-paranormal for $p>0$, if

$$
\left\||T|^{p} U|T|^{p} x\right\|\|x\| \geq\left\|\left.T\right|^{p} x\right\|^{2}
$$

holds for all $x \in H$, where $U$ is the partial isometry appearing in the polar decomposition $T=U|T|$ of $T$ with $|T|=\left(T^{*} T\right)^{1 / 2}$.

For $p>0$, an operator $T \in B(H)$ is of class $A(p, p)$ if it satisfies an operator inequality

$$
\left(\left|T^{*}\right|^{p}|T|^{2 p}\left|T^{*}\right|^{p}\right)^{1 / 2} \geq\left|T^{*}\right|^{2 p} .
$$

For $p, q>0$, an operator $T \in B(H)$ is of class $A(p, q)$ if it satisfies an operator inequality

$$
\left(\left|T^{*}\right|^{q}|T|^{2 p}\left|T^{*}\right|^{q}\right)^{q / p+q} \geq\left|T^{*}\right|^{2 q}
$$

In this sequel we introduce ${ }^{*} p$-paranormal operator, classes of operators ${ }^{*} A(p, p)$ for $p>0$ and ${ }^{*} A(p, q)$ for $p, q>0$ as follows:

A $p$-hyponormal operator is ${ }^{*} p$-paranormal if

$$
\left\||T|^{p} U|T|^{p} x\right\|\|x\| \geq\left\|\left|T^{*}\right|^{p} x\right\|^{2} .
$$

For $p>0$ a $p$-hyponormal operator $T \in{ }^{*} A(p, p)$ if it satisfies an operator inequality

$$
|T|^{2 p} \geq\left(|T|^{p}\left|T^{*}\right|^{2 p}|T|^{p}\right)^{1 / 2} .
$$

More generally, we define the class ${ }^{*} A(p, q)$ for $p, q$ 
$>0$ by an operator inequality

$$
|T|^{2 q} \geq\left(|T|^{q}\left|T^{*}\right|^{2 p}|T|^{q}\right)^{q / p+q} .
$$

Remark (2.1). If $T$ is $p$-hyponormal then using Furuta inequality $(1.1)(\S 1)$ it can be proved easily that $T \in A(p, p)$.

Remark (2.2). By inequality (2.6) we have

$$
\begin{aligned}
& \{p \text {-hyponormal, } 0<p<1\} \\
& \subset\left\{\text { class }^{*} A(p, q) ; p, q \in(0,1)\right\} .
\end{aligned}
$$

The well known theorem of $T$. Ando [3] for paranormal operator is required in the proof of our main result.

Theorem (2.3). (Ando's Theorem): An operator $T$ is paranormal if and only if

$$
T^{*^{2}} T^{2}+2 k T^{*} T+k^{2} \geq 0
$$

for all real $k$.

\section{Main Results}

M. Fujii, et al. [1] proved the following theorem [1; Theorem 3.4].

Theorem (3.1). If $T \in A(p, p)$ for $p>0$ then $T$ is p-paranormal.

In the following first we present an alternative way in which Theo (3.1) is proved in [1]. For this we have considered a quadratic form analogous to inequation (2.7) (\$2). We also present a necessary and sufficient condition for a p-hyponormal operator $T$ to be a ${ }^{*} p$-paranormal operator and the monotonicity of class ${ }^{*} A(p, q)$.

Theorem (3.2). A $p$-hyponormal operator $T \in B(H)$ is $p$-paranormal if and only if $|T|^{4 p}+2 k|T|^{2 p}+k^{2} \geq 0$ for all $k \in R$ and $p>0$.

Proof. Let $\mathrm{T}=U|T|$ be $p$-hyponormal where $U$ is partial isometry, hence

$$
U|T|^{p}=\left|T^{*}\right|^{p} U \text { and } U^{*}\left|T^{*}\right|^{p}=|T|^{p} U^{*} .
$$

We have

$$
\begin{aligned}
U|T|^{2 p} & =U|T|^{p}|T|^{p}=\left|T^{*}\right|^{p} U|T|^{p} \\
& =\left|T^{*}\right|^{p}\left|T^{*}\right|^{p} U=\left|T^{*}\right|^{2 p} U
\end{aligned}
$$

and

$$
\begin{aligned}
U^{*}\left|T^{*}\right|^{2 p} & =U^{*}\left|T^{*}\right|^{p}\left|T^{*}\right|^{p}=|T|^{p} U^{*}\left|T^{*}\right|^{p} \\
& =|T|^{p}|T|^{p} U^{*}=|T|^{2 p} U^{*} .
\end{aligned}
$$

Now,

$$
|T|^{4 p}+2 k|T|^{2 p}+k^{2} \geq 0
$$

for all $k \in R$

$$
\Leftrightarrow\left(\left(|T|^{4 p}+2 k|T|^{2 p}+k^{2}\right) x, x\right) \geq 0
$$

for all $k \in R$

$$
\Leftrightarrow\left(|T|^{4 p} x, x\right)+2 k\left(|T|^{2 p} x, x\right)+k^{2}(x, x) \geq 0
$$

for all $k \in R$

$$
\Leftrightarrow\left\|\left.T\right|^{2 p} x\right\|^{2}+2 k\left\|\left.T\right|^{p} x\right\|^{2}+k^{2}\|x\|^{2} \geq 0
$$

for all $k \in R$.

We know that if $a>0, b$ and $c$ are real numbers then $a t^{2}+2 b t+c \geq 0$ for every real $t$ if and only if $b^{2}-4 a c \leq 0$. Hence

$$
|T|^{4 p}+2 k|T|^{2 p}+k^{2} \geq 0
$$

for all $k \in R$

$$
\begin{aligned}
& \Leftrightarrow 4\left\||T|^{p} x\right\|^{4} \leq 4\left\||T|^{2 p} x\right\|^{2}\|x\|^{2} \\
& \Leftrightarrow\left\|\left.T\right|^{p} x\right\|^{4} \leq\left\||T|^{2 p} x\right\|^{2}\|x\|^{2} \\
& =\left(|T|^{2 p} x,|T|^{2 p} x\right)\|x\|^{2} \\
& =\left(|T|^{2 p} x, U^{*} U|T|^{2 p} x\right)\|x\|^{2} \\
& =\left(U|T|^{2 p} x, U|T|^{2 p} x\right)\|x\|^{2} \\
& =\left(U|T|^{2 p} x,\left|T^{*}\right|^{2 p} U x\right)\|x\|^{2} \\
& \left(\because U|T|^{2 p}=\left|T^{*}\right|^{2 p} U\right) \\
& =\left(U^{*}\left|T^{*}\right|^{2 p} U|T|^{2 p} x, x\right)\|x\|^{2} \\
& =\left(U^{*}\left|T^{*}\right|^{2 p}\left|T^{*}\right|^{2 p} U x, x\right)\|x\|^{2} \\
& =\left(U^{*}\left|T^{*}\right|^{4 p} U x, x\right)\|x\|^{2} .
\end{aligned}
$$

Since $T$ be $p$-hyponormal, by Remark (2.1) (\$2) $T \in A(p, p)$ i.e.

$$
\begin{aligned}
\left|T^{*}\right|^{2 p} & \leq\left(\left|T^{*}\right|^{p}|T|^{2 p}\left|T^{*}\right|^{p}\right)^{1 / 2} \\
& \Rightarrow\left|T^{*}\right|^{4 p} \leq\left|T^{*}\right|^{p}|T|^{2 p}\left|T^{*}\right|^{p} .
\end{aligned}
$$

Hence

$$
\begin{aligned}
\left\||T|^{p} x\right\|^{4} & \leq\left(U^{*}\left|T^{*}\right|^{4 p} U x, x\right)\|x\|^{2} \\
& \Leftrightarrow\left\||T|^{p} x\right\|^{4} \leq\left(U^{*}\left|T^{*}\right|^{p}|T|^{2 p}\left|T^{*}\right|^{p} U x, x\right)\|x\|^{2} \\
& =\left(|T|^{p} U^{*}|T|^{2 p} U|T|^{p} x, x\right)\|x\|^{2}
\end{aligned}
$$




$$
\begin{aligned}
& {\left[\because U^{*}\left|T^{*}\right|^{p}=|T|^{p} U^{*} \text { and }\left|T^{*}\right|^{p} U=U|T|^{p}\right]} \\
& =\left(\left(|T|^{p} U^{*}|T|^{p}\right)\left(|T|^{p} U|T|^{p}\right) x, x\right)\|x\|^{2} \\
& =\left(|T|^{p} U|T|^{p} x,|T|^{p} U|T|^{p} x\right)\|x\|^{2} \\
& =\left\|\left.\left.T\right|^{p} U|T|^{p} x\right|^{2}\right\| x \|^{2} \\
& \Leftrightarrow\left\|\left.T\right|^{p} x\right\|^{2} \leq\left\||T|^{p} U|T|^{p} x\right\|\|x\|
\end{aligned}
$$

i.e. if and only if $T$ is $p$-paranormal.

Remark (3.3). Theorem (3.2) is independent of $x \in H$ being taken as unit vector where as M. Fujii, et al. [1] have considered $\boldsymbol{x} \in H$ as unit vector in the result [1, Theo. 3.4].

The following result presents a necessary and sufficient condition for $p$-hyponormal operator $T$ to be a ${ }^{*} p$ paranormal operator.

Theorem (3.4). A $p$-hyponormal operator $T$ is ${ }^{*} p$ paranormal if and only if

$$
|T|^{4 p}+2 k\left|T^{*}\right|^{2 p}+k^{2} \geq 0 \text { for all } k \in R .
$$

Proof. Let $T=U|T|$ be $p$-hyponormal operator where $U$ is a partial isometry also let $S=U|T|^{p}$ so that $U|T|^{p}=\left|T^{*}\right|^{p} U, U^{*}\left|T^{*}\right|^{p}=|T|^{p} U^{*}, U|T|^{2 p}=\left|T^{*}\right|^{2 p} U$ and $U^{*}\left|T^{*}\right|^{2 p}=|T|^{2 p} U^{*}$. Now

$$
|T|^{4 p}+2 k\left|T^{*}\right|^{2 p}+k^{2} \geq 0
$$

for all $k \in R$

$$
\Leftrightarrow\left(|T|^{4 p} x, x\right)+2 k\left(\left|T^{*}\right|^{2 p} x, x\right)+k^{2}(x, x) \geq 0
$$

for all $k \in R$

$$
\Leftrightarrow\left\|\left.\left|T^{2 p} x\left\|^{2}+2 k\right\|\right| T^{*}\right|^{p} x\right\|^{2}+k^{2}\|x\|^{2} \geq 0
$$

for all $k \in R$

$$
\begin{aligned}
& \Leftrightarrow\left\|\left.T^{*}\right|^{p} x\right\|^{4} \leq\left\|\left.T\right|^{2 p} x\right\|^{2}\|x\|^{2} \\
& =\left(|T|^{2 p} x,|T|^{2 p} x\right)\|x\|^{2} \\
& =\left(U|T|^{2 p} x, U|T|^{2 p} x\right)\|x\|^{2} \\
& =\left(U|T|^{2 p} x,\left|T^{*}\right|^{2 p} U x\right)\|x\|^{2} \\
& =\left(U^{*}\left|T^{*}\right|^{2 p} U|T|^{2 p} x, x\right)\|x\|^{2} \\
& =\left(U^{*}\left|T^{*}\right|^{2 p}\left|T^{*}\right|^{2 p} U x, x\right)\|x\|^{2}
\end{aligned}
$$

i.e., $\quad\left\|\left.T^{*}\right|^{p} x\right\|^{4} \leq\left(U^{*}\left|T^{*}\right|^{4 p} U x, x\right)\|x\|^{2}$.
Since $T$ is $p$-hyponormal so $T \in A(p, p)$, i.e.

$$
\begin{array}{ll} 
& \left(\left|T^{*}\right|^{p}|T|^{2 p}\left|T^{*}\right|^{p}\right)^{1 / 2} \geq\left|T^{*}\right|^{2 p} \\
\text { i.e. } \quad & \left|T^{*}\right|^{4 p} \leq\left|T^{*}\right|^{p}|T|^{2 p}\left|T^{*}\right|^{p} .
\end{array}
$$

From (3.2) and (3.3), we have

$$
|T|^{4 p}+2 k\left|T^{*}\right|^{2 p}+k^{2} \geq 0
$$

for all $k \in R$

$$
\begin{aligned}
& \Leftrightarrow\left\|\left.T^{*}\right|^{p} x\right\|^{4} \leq\left(U^{*}\left|T^{*}\right|^{p}|T|^{2 p}\left|T^{*}\right|^{p} U x, x\right)\|x\|^{2} \\
& =\left(|T|^{p} U^{*}|T|^{2 p} U|T|^{p} x, x\right)\|x\|^{2} \\
& =\left(\left(|T|^{p} U^{*}|T|^{p}\right)\left(|T|^{p} U|T|^{p}\right) x, x\right)\|x\|^{2} \\
& =\left(|T|^{p} U|T|^{p} x,|T|^{p} U|T|^{p} x\right)\|x\|^{2} \\
& =\left\||T|^{p} U|T|^{p} x\right\|^{2}\|x\|^{2} \\
& \Leftrightarrow\left\|\left.T^{*}\right|^{p} x\right\|^{2} \leq\left\|\left.T\right|^{p} U|T|^{p} x\right\|\|x\|
\end{aligned}
$$

i.e. if and only if $T$ is ${ }^{*} p$-paranormal.

In the following we present monotonicity of " $A(p, q)$. We need Furuta inequality $[2,4]$ to prove the following theorem, see also $[5,6]$.

Theorem (3.5). If $0<p<p^{\prime}$ and $0<q$ then

$$
{ }^{*} A(q, p) \subseteq \subseteq^{*} A\left(q, p^{\prime}\right) \text {. }
$$

Proof. Let $T \in^{*} A\left(t, s^{\prime}\right)$ where $0<s^{\prime}<s$ and $0<t$ then by the definition of class ${ }^{*} A(p, q)$ for $p, q>0$.

$$
A=|T|^{2 s^{\prime}} \geq\left(|T|^{s^{\prime}}\left|T^{*}\right|^{2 t}|T|^{s^{\prime}}\right)^{s^{\prime} /\left(s^{\prime}+t\right)}=B
$$

We apply it to (1.2) (§1), in the case when $r_{0}=\frac{s-s^{\prime}}{2 s^{\prime}}$, $p_{0}=\frac{s^{\prime}+t}{s^{\prime}}, \quad q_{0}=\frac{s+t}{s}$. We have

$$
\left(1+2 r_{0}\right) q_{0}=\left(1+\frac{s-s^{\prime}}{s^{\prime}}\right)\left(\frac{s+t}{s}\right)=\frac{s}{s^{\prime}} \frac{(s+t)}{s}=\frac{s+t}{s^{\prime}}
$$

and

$$
p_{0}+2 r_{0}=\frac{s^{\prime}+t}{s^{\prime}}+\frac{s-s^{\prime}}{s^{\prime}}=\frac{s^{\prime}+t+s-s^{\prime}}{s^{\prime}}=\frac{s+t}{s^{\prime}} .
$$

Hence $\left(1+2 r_{0}\right) q_{0}=p_{0}+2 r_{0}$, so that

$$
\begin{aligned}
& {\left[|T|^{\frac{2 s^{\prime}\left(s-s^{\prime}\right)}{2 s^{\prime}}}|T|^{\frac{2 s^{\prime}\left(s^{\prime}+t\right)}{s^{\prime}}}|T|^{\frac{2 s^{\prime}\left(s-s^{\prime}\right)}{2 s^{\prime}}}\right]^{s /(s+t)}} \\
& \geq\left[|T|^{\frac{2 s^{\prime}\left(s-s^{\prime}\right)}{2 s^{\prime}}}\left(|T|^{s^{\prime}}\left|T^{*}\right|^{2 t}|T|^{s^{\prime}}\right)^{\frac{s^{\prime} s^{\prime}+t+t}{s^{\prime}}}|T|^{\frac{2 s^{\prime}\left(s-s^{\prime}\right)}{2 s^{\prime}}}\right]^{s /(s+t)}
\end{aligned}
$$


i.e. $|T|^{2 s} \geq\left(|T|^{s-s^{\prime}+s^{\prime}}\left|T^{*}\right|^{2 t}|T|^{s^{\prime}+s-s^{\prime}}\right)^{s /(s+t)}$

i.e. $|T|^{2 s} \geq\left(|T|^{s}\left|T^{*}\right|^{2 t}|T|^{s}\right)^{s /(s+t)}$

i.e. $T \in{ }^{*} A(t, s)$.

Hence

$$
{ }^{*} A\left(t, s^{\prime}\right) \subseteq{ }^{*} A(t, s)
$$

\section{REFERENCES}

[1] M. Fujii, D. Jung, S. H. Lee, M. Y. Lee and R. Nakamoto, "Some Classes of Operators Related to Paranormal and Log-Hyponormal Operators," Japanese Journal of Mathematics, Vol. 51, No. 3, 2000, pp. 395-402.
[2] T. Furuta, " $A \geq B \geq 0$ Assures $\left(B^{r} A^{p} B^{r}\right)^{1 / q} \geq B^{(p+2 r) / q}$ for $r \geq 0, \quad p \geq 0, \quad q \geq 1$ with $(1+2 r) q \geq p+2 r$," Proceedings of the American Mathematical Society, Vol. 101, No. 1, 1987, pp. 85-88. doi:10.2307/2046555

[3] T. Ando, "Operators with a Norm Condition," Acta Scientiarum Mathematicarum, Vol. 33, 1972, pp. 169-178.

[4] T. Furuta, "Elementary Proof of an Order Preserving Inequality," Proceedings of the Japan Academy, Vol. 65, No. 5, 1989, p. 126. doi:10.3792/pjaa.65.126

[5] M. Fujii, "Furuta's Inequality and Its Mean Theoretic Approach," Journal of Operator Theory, Vol. 23, No. 1, 1990, pp. 67-72.

[6] E. Kamei, “A Satellite to Furuta's Inequality,” Japanese Journal of Mathematics, Vol. 33, 1988, pp. 883-886. 